\title{
Hug behavior response model for generation of hug behavior with humans
}

\author{
Mitsuru JINDAI*, Shunsuke OTA*, Toshiyuki YASUDA* and Tohru SASAKI* \\ ${ }^{*}$ Graduate School of Science and Engineering, University of Toyama \\ 3190, Gofuku, Toyama, Toyama, 930-8555, Japan \\ E-mail: jindai@eng.u-toyama.ac.jp
}

Received: 4 August 2017; Revised: 1 February 2018; Accepted: 18 March 2018

\begin{abstract}
In human face-to-face communication, embodied sharing using the synchronization of embodied rhythms is promoted by embodied interactions. Therefore, embodied interactions are critical for smoothly initiating coexistence and communication. In particular, hug behavior can effectively promote the synchronization of embodied rhythms, being one of the types of embodied interactions wherein humans have whole-body contact with each other. In the case of a human and a robot, it is likely that the robot could effectively synchronize an embodied rhythm with a human using hug behavior. In the authors' previous study, a behavior model for the generation of hug behavior with humans was proposed. Furthermore, the timing of a human's voice greeting was found to be critical to the generation of smooth arm motion by a robot. However, this model generates only arm motions for hugs. It is not capable of generating an overall flow of hug behavior. Therefore, this study proposes a hug behavior response model that generates response behaviors when humans request hugs while approaching the robot. Furthermore, a hug robot system that uses the proposed model is developed. The effectiveness of the proposed hug behavior response model is demonstrated by sensory evaluations using the robot system.
\end{abstract}

Keywords : Hug behavior, Embodied interaction, Hug robot system, Human-robot interaction, Analysis by synthesis, Behavioral analysis

\section{Introduction}

In human face-to-face communication, embodied sharing using the synchronization of embodied rhythms is promoted by embodied interactions. Therefore, embodied interactions are critical to the smooth initiation of coexistence and communication. In the case of a human and a robot, the robot can begin to communicate and coexist smoothly with the human without producing feelings of aversion against the human if the robot generates an embodied interaction that is emotionally acceptable to the human. A robot that can generate such an interaction is emotionally acceptable to humans because it produces feelings of security (Rani, et al., 2007). Embodied interactions that involve direct contact are considered to synchronize the embodied rhythms particularly effectively.

Hug behavior is an embodied interaction that involves direct contact, in which humans achieve whole-body contact with each other. Therefore, hug behaviors are likely to synchronize embodied rhythms effectively. In the case of a human and a robot, the robot can effectively synchronize the embodied rhythms by hug behavior. Embodied interactions between humans and robots that involve direct contact have been discussed (Watanabe, et al., 2012)(Jindai, et al., 2011). In particular, hug behavior between humans and robots have been examined in previous studies (Nakanishi, et al., 2013)(Tsetserukou, et al., 2010)(Teh, et al., 2008)(DiSalvo, et al., 2003). These studies examined remote hug communications between partners and shapes of hugging robots. However, these studies did not discuss behavior models for the generation of hug interaction with humans. Thus, in the authors' previous study, arm motions of hugs between humans were analyzed, and a behavior model was proposed for the generation of hug behavior with a human (Jindai, et al., 2016). Furthermore, a hug robot system that uses the proposed model was developed. Moreover, in hug behavior between a human and a robot, the timing of the human's voice greeting was found to be critical, by analysis-by-synthesis using the 
developed hug robot system, to the generation of smooth arm motion. However, this model generates only arm motions for hugs. It is not capable of generating an overall flow of hug behavior.

Therefore, in this study, a hug behavior response model is proposed for the generation of hug behavior with humans who approach a robot. This model generates a hug response behavior when humans request hugs while approaching the robot. In this model, the hug response behavior is generated based on an analysis of hug behavior between humans. Thus, it is likely that the generated hug behavior is emotionally acceptable to a human. Furthermore, a hug robot system that uses the proposed model was developed. Subsequently, using the developed robot system, the timing of the beginning of arm motion and voice greeting, preferred by humans, were assessed by sensory evaluations. Furthermore, the effectiveness of the proposed hug behavior response model is demonstrated.

\section{Analysis of Hug Behavior between Humans}

\subsection{Hug Experiment between Humans}

Hug behavior between humans was measured experimentally to provide a foundation for modeling a similar behavior between a human and a robot.

During the experiment, human behavior was measured using a three-dimensional motion capture system. The request-side and response-side were determined beforehand. The research participants were told that the request-side will request hug behavior from the response-side. Eleven reflection markers (head, both shoulders, both elbows, both wrists, both hands, and both ankles) were attached to the research participants on either side. The positions of the markers were tracked by ten cameras at a sampling rate of $120 \mathrm{~Hz}$, with an accuracy of $\pm 1 \mathrm{~mm}$. Furthermore, microphones were used to record the voice greetings of either side. Figure 1 illustrates a typical experimental scenario. The research participants are initially facing each other at a distance of $3000 \mathrm{~mm}$ with their arms extended downwards. The request-side research participant approaches the response-side research participant and starts to move his or her arms at an arbitrary time in order to hug the response-side research participant. Consequently, the response-side research participant moves his or her arms in response to this behavior by the request-side research participant. The research participants were twenty healthy students (ten pairs) aged between 20 and 24, and each pair hugged ten times.

All experiments were conducted with the approval of the Ethical Review Committee of University of Toyama.

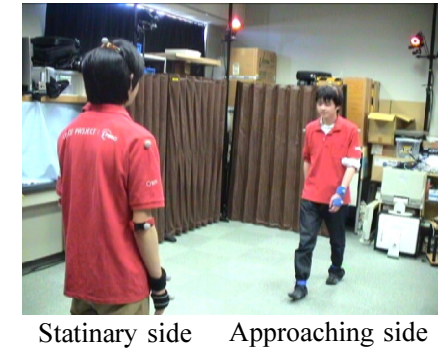

Approaching behavior

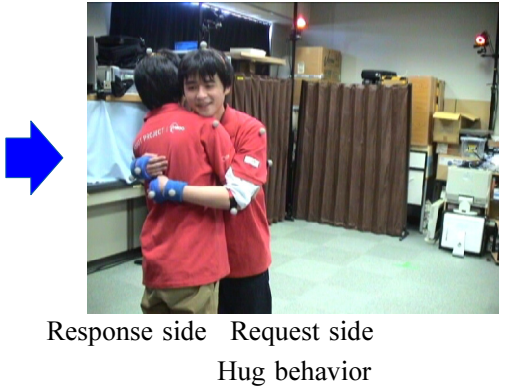

Hug behavior

Fig. 1 Experimental scenario of hug behavior between humans

\subsection{Analysis Results of Hug Behavior between Humans}

For the generation of hug behavior, a hug behavior generation model, which is described later, has already been proposed based on analysis of hug behaviors between humans in the authors' previous study (Jindai, et al., 2016). Furthermore, in this previous paper, a timing of initiation of a robot's arm motion were assessed by sensory evaluations during hug behavior between a request-side human and a response-side robot. As the result, the timing of human's voice greetings when compared to arm motions was found to be critical to the generation of smooth arm motion by a robot. When a robot is on the response-side, it has to generate its hug behavior in response to a voice greeting by a human. Thus, this study measured the time intervals between the voice greetings of the request-sides to the arm motions and voice greetings of the response-sides. Furthermore, in order to analyze the hug behavior of the response-side, the time interval between the arm motions and voice greetings of the response-side were measured.

2.2.1. Time intervals between voice greeting of request-side and arm motion of response-side The time intervals between the initiation of the voice greeting of the request-side and the beginning of the arm motion of the response-side, during human hug behavior, were recorded. The time intervals were recorded for 10 hug behaviors, performed by each of the twenty pairs (200 records in total). Figure 2 illustrates a histogram from $-0.1 \mathrm{~s}$ to $1.3 \mathrm{~s}$. This range includes 
$90 \%$, or more, of the research participants. The arm motion of the request-side begins prior to the arm motion of the response-side, where the time interval is positive. Furthermore, it can be observed that the majority of the response-side research participants began their arm motions $0.3 \mathrm{~s}$ after the initiation of the voice greeting by the request-side research participants.

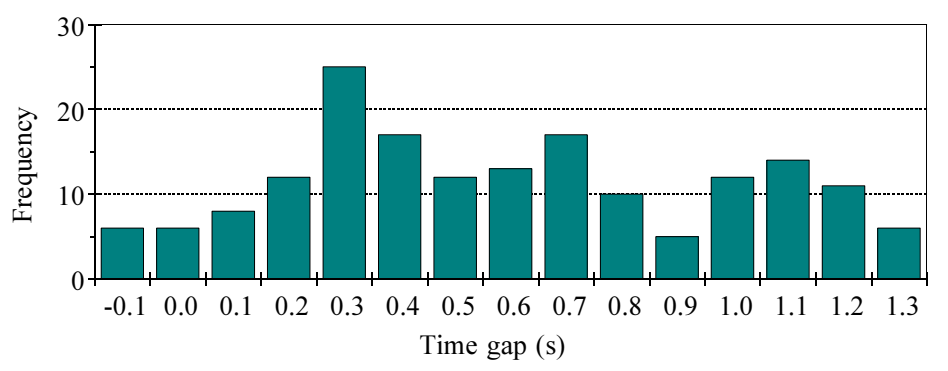

Fig. 2 Time intervals between voice greetings of request-side and arm motion of response-side

2.2.2. Time intervals between voice greetings request-side and response-side The time intervals between the initiation of the voice greetings by the request-side and the response-side research participants were also recorded. Figure 3 illustrates a histogram of the time intervals. This range includes $90 \%$, or more, of the research participants. As shown in this figure, the time interval, measured for the majority of the research participants, was widely distributed between $0.4 \mathrm{~s}$ and $1.8 \mathrm{~s}$, with a peak at $0.6 \mathrm{~s}$. A positive time interval indicates that the voice greeting by the response-side began after the voice greeting by the request-side. The majority of the response-side research participants began their voice greetings $0.6 \mathrm{~s}$ after the initiation of the voice greetings by the request-side research participants.

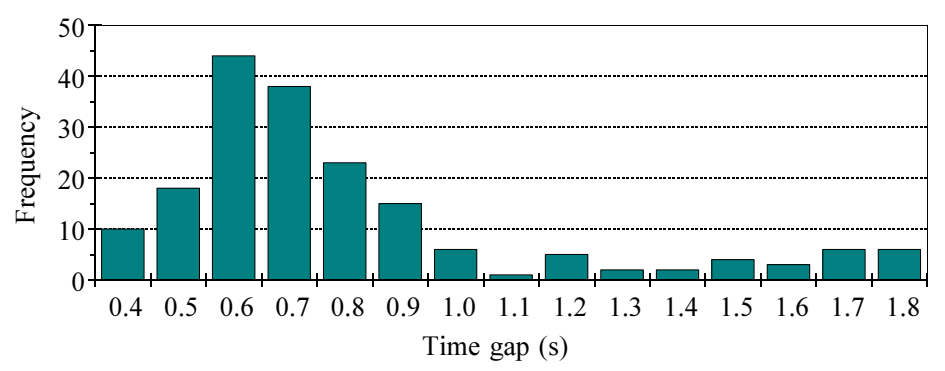

Fig. 3 Time intervals between voice greetings of request-side and response-side

2.2.3. Time intervals between arm motion and voice greeting of response-side For analyzing the relationships between the arm motions and voice utterances of the response-side, the time intervals between the beginning of arm motions and the initiation of voice greetings by the response-side were recorded. Figure 4 illustrates a histogram of the time intervals. This range includes $85 \%$, or more, of the research participants. The figure shows that the time intervals measured for the majority of the research participants were widely distributed between $-0.6 \mathrm{~s}$ and $0.8 \mathrm{~s}$, with a peak at 0.1 s. A positive time interval indicates that the voice greeting began after the arm motion. The majority of the response-side research participants began their voice greetings $0.1 \mathrm{~s}$ after the beginning of the arm motions.

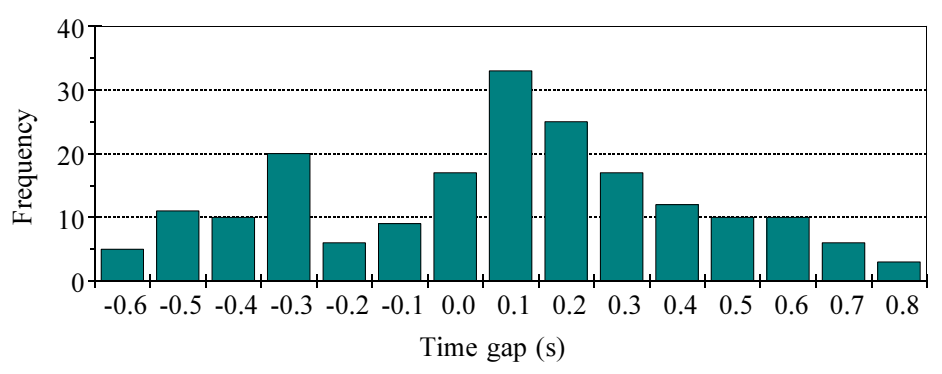

Fig. 4 Time intervals between arm motion and voice greeting of response-side

The above analysis reveals that a majority of the response-side humans began their arm motions $0.3 \mathrm{~s}$ after the initiation of the voice greeting by the request-side humans. Furthermore, the majority of the response-side humans began their voice greetings $0.1 \mathrm{~s}$ after the beginning of the arm motions. From these results, it can be inferred that the majority of the response-side humans utter a voice greeting $0.4 \mathrm{~s}$ after the initiation of a voice greeting by the request-side 
humans when arm motion is used as reference. However, this time does not correspond with the $0.6 \mathrm{~s}$, which is the time interval between the voice greetings by the request-side and those by the response-side, described in the above analysis. Therefore, it is indicated that the time intervals between the voice greetings by the request-side and response-side humans vary depending on the reference.

\section{Hug Behavior Response Model}

This study proposes a hug behavior response model that generates hug response behavior when humans request hugs while approaching the robot. The model begins the arm motion and utterance of voice greeting according to the request motions of humans in order to generate an overall flow of hug behavior between a human and a robot.

\subsection{Beginning of Arm motion and Voice Greeting}

In the authors' previous study, it has been discussed that a robot should move its arms in response to a human's voice greeting when the robot is on the response-side in a hug interaction between a human and a robot (Jindai, et al., 2016). Furthermore, the timing of a human's voice greeting was found to be critical for the generation of smooth hug behavior by a robot. Based on these concepts, in this model, a robot begins its arm motion and utterance of a voice greeting according to the voice greetings uttered by humans.

\subsection{Generation of Arm Motion Using Hug Behavior Generation Model}

A hug behavior generation model for the generation of arm motion has already been proposed by the authors (Jindai, et al., 2016). From the analysis of behavior between humans, it was observed that the velocity patterns of human arm joint movements during hug behavior are bell-shaped and wave-shaped. Therefore, this model generates arm motions for hug behavior by the movement of arm joints, which are generated by these two velocity patterns. In this model, the rotational motion of shoulder, elbow, forearm, and wrist joints are generated using the bell-shaped velocity pattern. In addition, the external and internal rotations of upper arms are generated using the wave-shaped velocity pattern. In order to produce right and left arm motions, similar to those of humans, the movement time, maximum velocity, peak time, and initial and target angles are matched to those of humans, as determined from the analysis of hug behavior between humans.

The generation of the bell-shaped and wave-shaped velocity patterns is described in the following sections.

3.2.1. Bell-Shaped Velocity Pattern A peak time of a bell-shaped velocity pattern is an important factor in order to generate hug behavior similar to those of humans, because arm joint movements are generated by this velocity pattern. Therefore, the hug behavior generation model uses a bell-shaped velocity pattern, which can be adjusted to the peak time.

The bell-shaped velocity pattern with an adjustable peak time is generated with the minimum jerk model as the foundation (Flash, et al., 1985). The minimum jerk model can accurately reproduce the point-to-point motion of human hands. In this model, the acceleration and velocity patterns exhibit smooth transitions. However, it is not feasible to adjust the peak time. Therefore, the two velocity patterns that are generated by the minimum jerk model are combined to adjust the peak time. The bell-shaped velocity pattern is generated as follows: First, two velocity patterns $V_{1}(t)$ and $V_{2}(t)$ are generated based on the minimum jerk model using Eq. (1). $T_{f}$ and $T_{p}$ denote the movement and peak times, respectively, of the target bell-shaped velocity pattern. $T_{p}$ is calculated from $T_{f}$ and $P$, which is the value of the peak time when $T_{f}$ is normalized to 1 . The maximum velocity $V_{\max }$ is calculated from the initial angle $R_{0}$ and the target angle $R_{f}$ using Eq. (2). Subsequently, the first half of the velocity pattern $V_{1}(t)$ and the latter half of the velocity pattern $V_{2}(t)$ are combined as illustrated in Fig. 5. As a result, the target bell-shaped velocity pattern is generated. In this pattern, the position and velocity vary smoothly, and it is feasible to adjust the peak time.

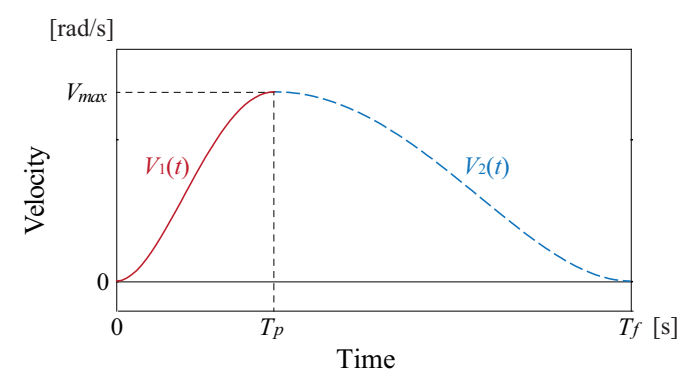

Fig. 5 Bell-shaped velocity pattern 


$$
\begin{aligned}
& V_{i}(t)=V_{\max } \frac{16}{T_{i}^{4}}\left(t^{4}-2 T_{i} t^{3}+T_{i}^{2} t^{2}\right) \quad(i=1,2) \\
& \text { Here, } T_{1}=2 P T_{f}, \quad T_{2}=2(1-P) T_{f}, \quad T_{p}=P T_{f} \\
& V_{\text {max }}=\frac{15}{8 T_{f}}\left(R_{f}-R_{0}\right)
\end{aligned}
$$

3.2.2. Wave-Shaped Velocity Pattern The wave-shaped velocity pattern is generated using a fifth-order curve and is illustrated in Fig. 6. Its shape is determined by the movement time, maximum velocity, and initial and target angles. The fifth-order curve is expressed by Eq. (3). The movement and peak times are denoted by $T_{f}$ and $T_{p}$, respectively and $T_{c}$ is the time at which the velocity becomes zero. The value of the constant $K$ is calculated from the initial angle $R_{0}$ and target angle $R_{f}$ using Eq. (4). The peak time $T_{p}$ is calculated using Eq. (5). Using these equations, $T_{c}$ is determined while the velocity at time $T_{p}$ attains its maximum value $V_{\max }$.

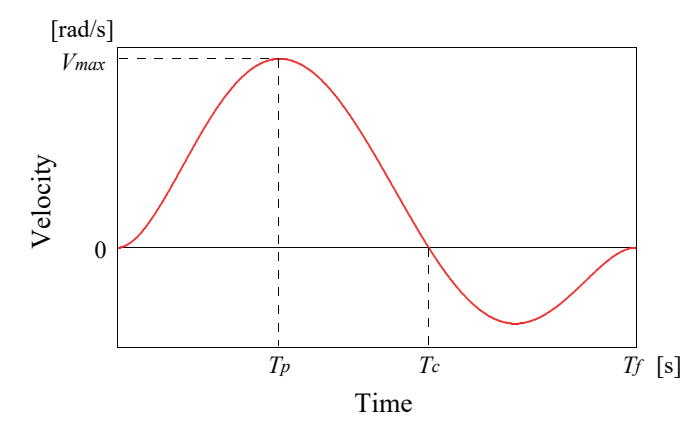

Fig. 6 Wave-shaped velocity pattern

$$
\begin{aligned}
& V(t)=K\left(T_{f}-t\right)^{2}\left(T_{c}-t\right) t^{2} \\
& K=\frac{60\left(R_{f}-R_{0}\right)}{2 T_{f}^{5} T_{c}-T_{f}^{6}} \\
& T_{p}=\frac{\left(4 T_{c}+3 T_{f}\right)-\sqrt{16 T_{c}^{2}-16 T_{f} T_{c}+9 T_{f}^{2}}}{10}
\end{aligned}
$$

\section{Hug Robot System}

A hug robot system that employs the proposed hug behavior response model was developed. The hug robot system is illustrated in Fig. 7. The robot system incorporates two arms. Each arm has seven degrees-of-freedom, three at the shoulder and one at the upper arm, elbow, forearm, and wrist, as illustrated in Fig. 8. The robot arms were fabricated based on the average size of a human arm (Kouchi, et al., 2000). Each joint of the robot was driven by a direct-current servomotor.

A magnetic sensor and a laser range finder were used to measure the positions of the human hands and the distance between the human and the robot. A microphone was used to input a voice greeting by a human, and the robot uttered a voice greeting by a speaker. The sampling time of the system was $5 \mathrm{~ms}$. The robot was capable of achieving the desired position accurately.

\section{Beginning of Arm Motion Experiment}

The hug behavior of the response-side begins according to the hug behavior of the request-side. Thus, it is considered that the beginning of the arm motion influences human emotions. Therefore, an experiment was performed with a human and a robot as the request-side and response-side, respectively, to determine the timing of the beginning of the arm motion preferred by humans. 


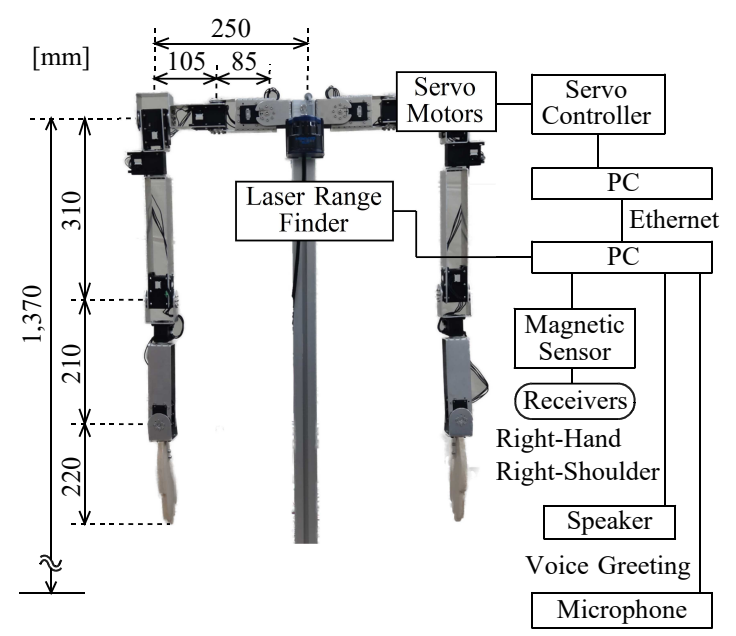

Fig. 7 Hug robot system

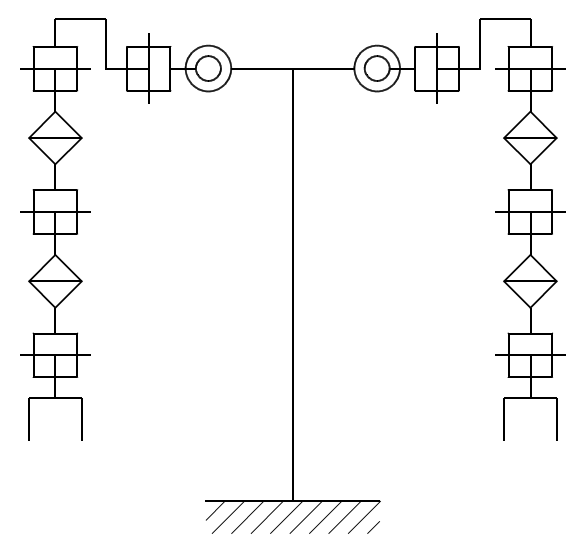

Fig. 8 Mechanism diagram

The experimental scenario is illustrated in Fig. 9. During the experiment, the research participant and the robot are initially facing each other at a distance of $3000 \mathrm{~mm}$. Consequently, the research participant approaches the robot and starts to move his or her arms at an arbitrary time, requesting hug behavior. In addition, the research participant utters a voice greeting during the hug response behavior. The robot then moves its arms according to this motion using the hug behavior response model. The robot generates hug behavior accompanied by a voice greeting.

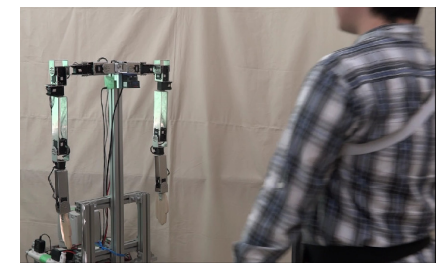

Approaching behavior

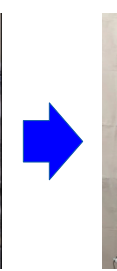

Fig. 9 Experimental scenario of hug behavior using robot system

\subsection{Experimental Method}

In the experiment described in the previous section, a number of humans on the response-side began their arm motions $0.3 \mathrm{~s}$ after the initiation of the voice greeting by the request-side. Therefore, three modes were used in this experiment, as shown in Fig. 10. In mode (a), the robot began its hug behavior simultaneously with a human's voice greeting. In modes (b) and (c), the robot began its hug behavior $0.3 \mathrm{~s}$ and $0.6 \mathrm{~s}$ after a human's voice greeting, respectively. The time interval is determined by the fact that the shortest time in which a large number of humans are capable of recognizing variation in movement is $0.3 \mathrm{~s}$. In this experiment, based on the analyses of hug behavior between humans, the robot generated hug behavior accompanied by a voice greeting that is uttered $0.1 \mathrm{~s}$ after the initiation of the robot's arm motion.

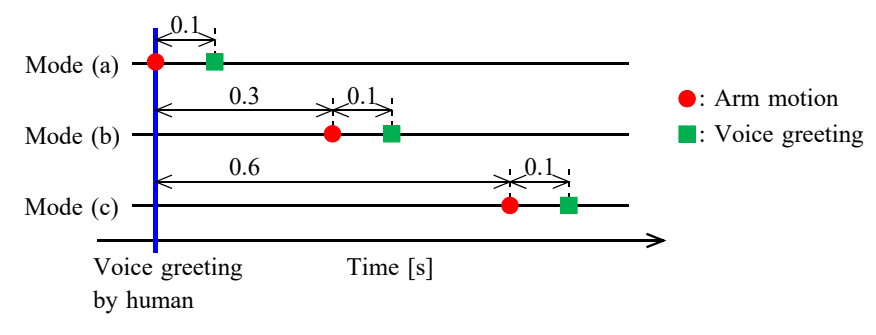

Fig. 10 Three modes in experiment

Prior to the sensory evaluation, the contents of the experiment and questionnaires were explained to the research participants. Furthermore, they practiced a hug with the robot using all modes for a sufficient amount of time. However, the differences between modes were not revealed to them. In the sensory evaluation, a paired comparison was first carried 
out and, subsequently, a seven-point bipolar rating was carried out. The research participants were thirty healthy students aged between 20 and 24 .

The paired comparison was performed for all combinations (six pairs) considering the order effect. The research participants were ordered to select the better mode comprehensively.

The seven-point bipolar rating with a scale from -3 (not at all) to 3 (extremely good), was determined for the following five items: "Ease of Hug," "Comfortable Velocity," "Security," "Politeness," and "Vitality." The research participants were ordered to provide evaluations for all items in each mode. Considering the order effect, each research participant evaluated all the modes in different orders, there were six different patterns for all combinations. Since there were thirty research participants, the six patterns were repeated five times throughout the whole experiment.

\subsection{Experimental Results}

5.2.1. Paired comparison The results of the paired comparison are illustrated in Table 1. The table illustrates the number of research participants that preferred the column mode to the row mode. The table indicates that mode (b) was preferred by the largest number of research participants. Furthermore, the Bradley-Terry model (Bradley, et al., 1952) was fitted to the results to analyze them quantitatively using Eq. (6).

$$
\begin{aligned}
& P_{i j}=\frac{\pi_{i}}{\pi_{i}+\pi_{j}} \\
& \sum_{i} \pi_{i}=\text { Const. }(=\text { Total }: 100)
\end{aligned}
$$

$\pi_{i}$ is the intensity of the preference for model $i . P_{i j}$ is the probability of the judgment that $i$ is better than $j$.

Using this model, the results of the paired comparison were expressed by the intensity of preference $\pi$, as illustrated in Fig. 11. The suitability of the model was validated by the goodness-of-fit and likelihood ratio tests. Mode (b) was rated as the most effective mode.

Table 1 Result of paired comparison

\begin{tabular}{|l|r|r|r|r|}
\hline & (a) & (b) & (c) & \multicolumn{1}{c|}{ Total } \\
\hline (a) & & 18 & 35 & 53 \\
\hline (b) & 42 & & 42 & 84 \\
\hline (c) & 25 & 18 & & 43 \\
\hline
\end{tabular}

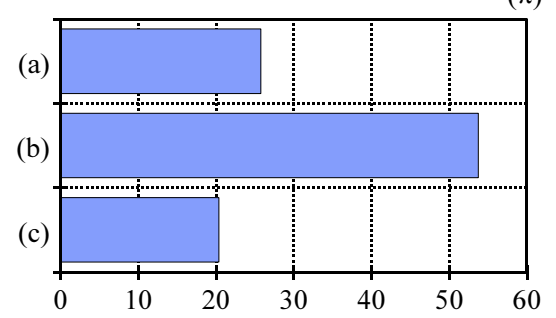

Fig. 11 Result of Bradley-Terry model

5.2.2. Seven-point bipolar rating The results of the seven-point bipolar rating are illustrated in Fig. 12. The markers indicate the mean value and the vertical bars indicate the standard deviation of each item. Furthermore, significant differences were estimated by the Friedman Test. Mode (b) was evaluated as being more effective than mode (a) with a significant difference of $5 \%$ for the item "Politeness." Furthermore, for the item "Vitality," mode (b) was evaluated as being more effective than mode (c) with a significant difference of $5 \%$. From these results, mode (b) was evaluated to be the most effective and this result is consistent with the result of the Bradley-Terry model.

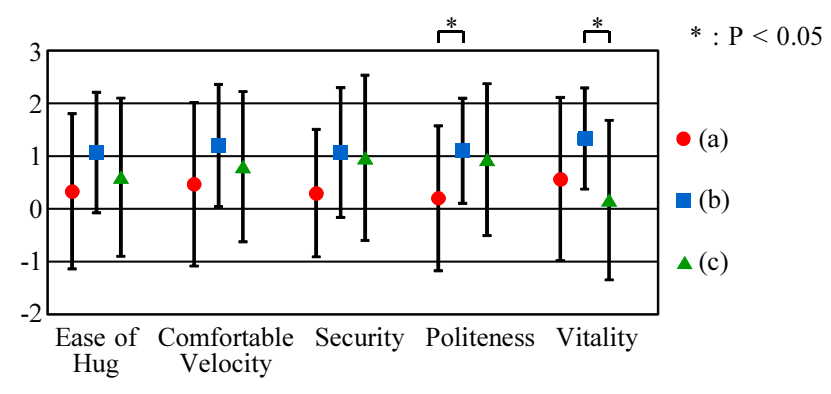

Fig. 12 Result of seven-point bipolar rating 
From the experimental results, mode (b) was determined to be the most preferred mode. Therefore, the hug behavior wherein the robot begins its arm motion $0.3 \mathrm{~s}$ after a voice greeting by a human is preferred by the majority of humans.

\section{Initiation of Voice Greeting Experiment}

In the analysis of hug behavior between humans, the majority of the response-side humans utter voice greetings 0.1 $\mathrm{s}$ after the beginning of their arm motions. Therefore, the majority of response-side humans utter a voice greeting $0.4 \mathrm{~s}$ after the initiation of a voice greeting by the request-side humans in the case where the response-side humans begin their arm motions $0.3 \mathrm{~s}$ after the initiation of a voice greeting by the request-side humans. In addition, the robot begins its arm motion $0.3 \mathrm{~s}$ after the initiation of a human's voice greeting, which is preferred by numerous humans in the above experiment. However, the majority of the response-side humans began their voice greeting $0.6 \mathrm{~s}$ after the initiation of voice greeting by the request-side humans. From these results, the majority of the response-side humans utter a voice greeting $0.4 \mathrm{~s}$ after the initiation of voice greeting by the request-side humans when the arm motion is used as reference. When the voice utterance is used as reference, the majority of the response-side humans utter a voice greeting $0.6 \mathrm{~s}$ after the initiation of the voice greeting by the request-side humans.

Therefore, experiments were performed to determine the timing of the voice utterance preferred by humans. Two experiments were conducted under varying conditions. The first condition is the case wherein the robot utters its voice greeting based on its arm motion. The other condition is the case wherein the robot utters its voice greeting based on human voice greetings. The experimental conditions remained similar to those described above.

\subsection{Experiment Based on Arm Motion}

6.1.1. Experimental Method The experiment was conducted under the condition wherein the robot utters a voice greeting based on its arm motion. The majority of the response-side humans utter a voice greeting $0.1 \mathrm{~s}$ after they begin move their arms. Furthermore, the shortest time in which a large number of humans are capable of recognizing a variation in the utterance of voice greeting is $0.3 \mathrm{~s}$. In addition, the robot begins its arm motion $0.3 \mathrm{~s}$ after the initiation of the human voice greeting from the above experimental results. Therefore, in this experiment, three modes were used as shown in Fig. 13. In mode (a), the robot utters a voice greeting $0.2 \mathrm{~s}$ before the beginning of its arm motion. In modes (b) and (c), the robot utters a voice greeting $0.1 \mathrm{~s}$ and $0.4 \mathrm{~s}$, respectively, after the beginning of its arm motion.

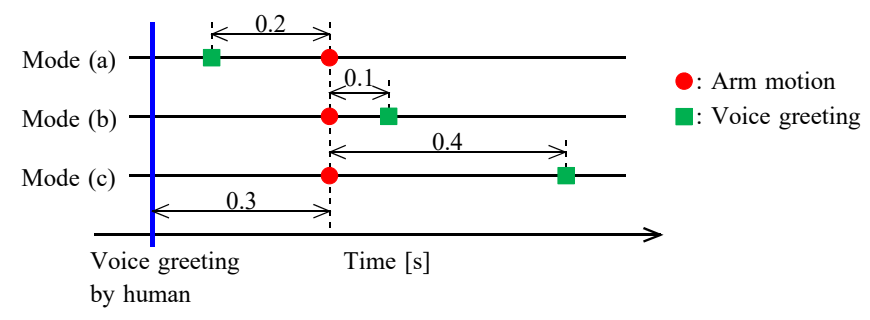

Fig. 13 Three modes in experiment

\subsubsection{Experimental Results}

\section{(1)Paired comparison}

The results of the paired comparison are illustrated in Table 2. Furthermore, the Bradley-Terry model was fitted to the results and the results of the Bradley-Terry model are illustrated in Fig. 14. The suitability of the model was validated by the goodness-of-fit and likelihood ratio tests, and mode (b) was found to be the most preferred mode. However, there was no variation between the evaluation of mode (b) and mode (c).

Table 2 Result of paired comparison

\begin{tabular}{|c|r|r|r|r|}
\hline & (a) & (b) & (c) & \multicolumn{1}{c|}{ Total } \\
\hline (a) & & 10 & 9 & 19 \\
\hline (b) & 50 & & 31 & 81 \\
\hline (c) & 50 & 29 & & 79 \\
\hline
\end{tabular}




\section{(2)Seven-point bipolar rating}

Figure 15 illustrates the results of the seven-point bipolar rating with significant differences estimated by the Friedman Test. Modes (b) and (c) were evaluated as being more effective than mode (a) with significant differences of $1 \%$ for the items "Comfortable Velocity" and "Security." For the items "Ease of Hug" and "Vitality," mode (b) was evaluated as being more effective than mode (a) with a significant difference of $5 \%$. Furthermore, mode (c) was evaluated as being more effective than mode (a) with a significant difference of $1 \%$ for the item "Politeness." However, there is no significant difference between modes (b) and (c). These results are consistent with the result of the Bradley-Terry model.

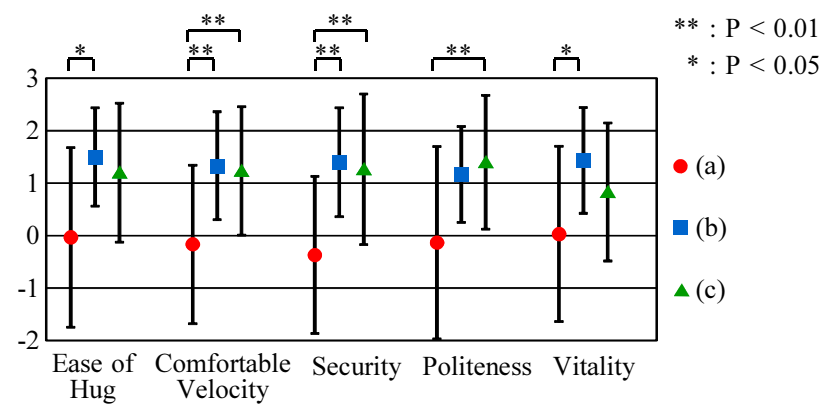

Fig. 15 Result of seven-point bipolar rating

From these results, modes (b) and (c) were evaluated to be equally effective. Humans prefer hug motions, which the robot utters $0.1 \mathrm{~s}$, or more, after the beginning of its arm motion. Therefore, humans approve the robot's utterance of voice greeting in a wide range when its arm motion is used as reference. Thus, there are individual variations in the preferred timing of utterance of voice greeting when the arm motion is used as reference.

\subsection{Experiment Based on Voice Greeting of Human}

6.2.1. Experimental Method The experiment was conducted under the condition wherein the robot utters its voice greeting based on the voice greetings of humans. The majority of the response-side humans began their voice greeting $0.6 \mathrm{~s}$ after the initiation of the voice greeting by the request-side humans. Furthermore, the shortest time in which a large number of humans are capable of recognizing a variation in the utterance of voice greeting is $0.3 \mathrm{~s}$. Therefore, in this experiment, three modes were used, as shown in Fig. 16. In modes (a), (b), and (c) the robot utters a voice greeting $0.3 \mathrm{~s}$, $0.6 \mathrm{~s}$, and $0.9 \mathrm{~s}$ after a voice greeting by the human, respectively. The robot begins its arm motion $0.3 \mathrm{~s}$ after the initiation of the human voice greeting from the above experimental results.

The experimental conditions remained similar to those of the experiments described above.

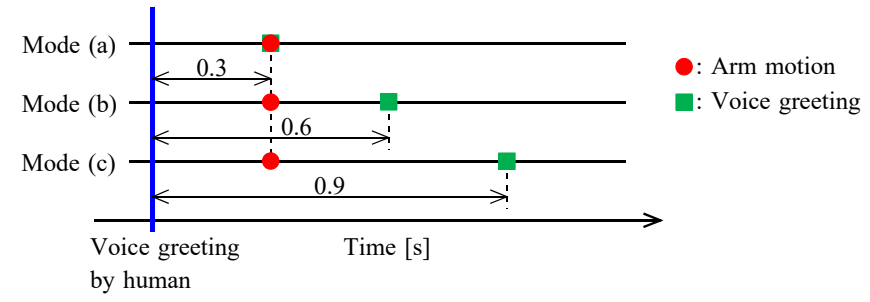

Fig. 16 Three modes in experiment

\subsubsection{Experimental Results}

\section{(1)Paired comparison}

The results of the paired comparison are presented in Table 3. Furthermore, the Bradley-Terry model was fitted to the results and the result of the Bradley-Terry model is illustrated in Fig. 17. The suitability of the model was validated by the goodness-of-fit and likelihood ratio tests and mode (b) was found to be the most preferred mode.

\section{(2)Seven-point bipolar rating}

The results of the seven-point bipolar rating are illustrated in Fig. 18. Modes (b) and (c) were evaluated as being more effective than mode (a) with significant differences of $1 \%$ and $5 \%$ for the item "Politeness." Mode (b) was evaluated as being more effective than mode (a) with a significant difference of $5 \%$ for the item "Ease of Hug." Furthermore, mode (c) was evaluated as being more effective than mode (a) with a significant difference of $1 \%$ for the item "Security." However, 
Table 3 Result of paired comparison

\begin{tabular}{|l|r|r|r|r|}
\hline & \multicolumn{1}{|c|}{ (a) } & (b) & (c) & \multicolumn{1}{c|}{ Total } \\
\hline (a) & & 15 & 31 & 46 \\
\hline (b) & 45 & & 42 & 87 \\
\hline (c) & 29 & 18 & & 47 \\
\hline
\end{tabular}

$(\pi)$

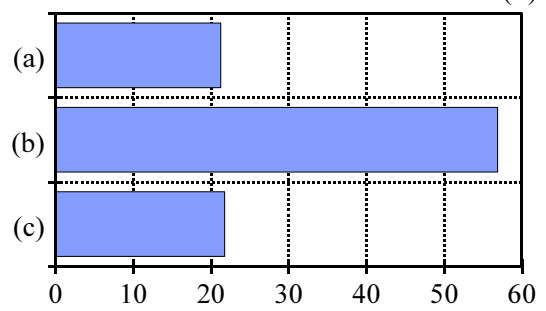

Fig. 17 Result of Bradley-Terry model

there is no significant difference between modes (b) and (c).

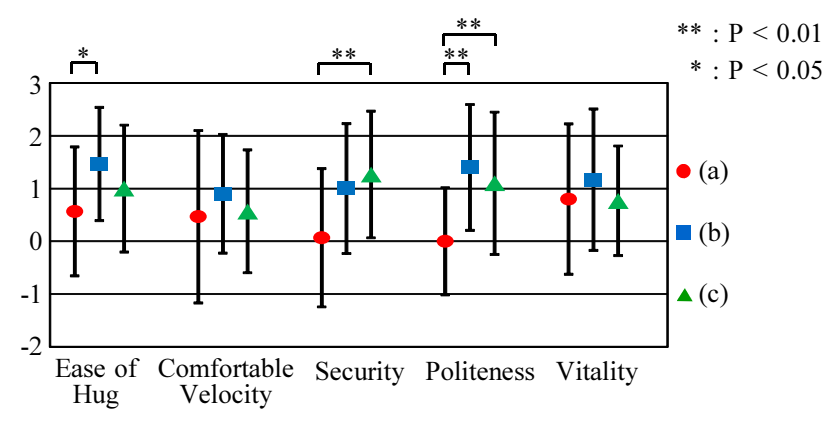

Fig. 18 Result of seven-point bipolar rating

In the seven-point bipolar rating, there is no variation between modes (b) and (c). However, mode (b) was evaluated as being more effective than mode (c) in the results of the Bradley-Terry model. Therefore, the most preferred mode is mode (b), wherein the robot utters a voice greeting $0.6 \mathrm{~s}$ after the human voice greeting. In addition, in the sevenpoint bipolar rating, all items in mode (b) were evaluated as positive. This indicates that hug behavior generated with the utterance of voice greetings as reference, is preferred by the majority of humans. Thus, the proposed hug behavior response model is capable of generating hug behavior that is preferred by the majority of humans and its effectiveness was demonstrated.

The results indicate that there are individual variations in the preferred timing of utterance of voice greeting when the arm motion is used as reference. However, when the voice utterance is used as reference, the individual variations in the preferred timing of utterance of voice greeting are reduced. Therefore, this indicates that the hug behavior wherein the robot utters its voice greeting based on the voice greetings of humans, is preferred by the majority of humans. Moreover, the hug behavior wherein arm motions and voice greetings begin $0.3 \mathrm{~s}$ and $0.6 \mathrm{~s}$, respectively, after the voice greeting of humans, is most highly evaluated.

\section{Conclusion}

In this study, a hug behavior response model was proposed for the generation of hug behavior for humans who approach a robot. This model generates a response behavior of a hug, when humans request hugs while approaching the robot. Furthermore, a hug robot system was developed that uses the proposed model. Subsequently, using the developed robot system, the timings of the beginning of the arm motion and voice greeting that are preferred by humans were assessed by sensory evaluations. In addition, the effectiveness of the proposed hug behavior response model was demonstrated.

The conclusions drawn from the experimental results are as follows:

- In the analyses of hug behavior between humans, the majority of the response-side humans began their voice greeting $0.6 \mathrm{~s}$ after the initiation of the voice greeting by the request-side humans. However, response-side humans utter a voice greeting $0.4 \mathrm{~s}$ after the initiation of voice greeting by the request-side humans when arm motion is used as reference. Therefore, it is indicated that the time intervals between voice greetings of the request-side and response-side humans vary depending on the reference.

- The individual variations have a wide range when the arm motion is used as reference. However, when the voice utterance is used as reference, individual variations in the preferred timing of utterance of voice greeting are reduced. Therefore, it is indicated that numerous humans prefer hug behavior in the case wherein the robot utters its voice greeting based on the voice greetings of humans. 
- In the sensory evaluation, the hug behavior wherein arm motions and voice greetings begin $0.3 \mathrm{~s}$ and $0.6 \mathrm{~s}$, respectively, after the human voice greeting, is most highly evaluated. Furthermore, the hug behavior is preferred by the majority of humans. This result indicates that the proposed hug behavior response model is capable of generating hug behavior, which is preferred by the majority of humans. Therefore, the effectiveness of the proposed model is demonstrated.

\section{Acknowledgments}

This work was supported by KAKENHI Grant Number 25330239 of the Japan Society for the Probehavior of Science (JSPS), Japan.

\section{References}

Bradley, WR. A. and Terry, M. E., Rank analysis of incomplete block designs, Biometrika, Vol.39 (1952), pp.324-345.

DiSalvo, C., Gemperle, F., Forlizzi, J. and Montgomery, E., The Hug: an Exploration of Robotic Form for Intimate Communication, Proceedings of 12th IEEE Workshop on Robot and Human Interactive Communication (2003), pp.403-408.

Flash, T. and Hogan, N., The Coordination of Arm Movements: An Experimentally Confirmed Mathematical Model, Journal of Neurosciences, Vol.5, No.7 (1985), pp.1688-1703.

Jindai, M. and Watanabe, T., Development of a Handshake Request Motion Model Based on Analysis of Handshake Motion between Humans, Proceedings of the 2011 IEEE/ASME International Conference on Advanced Intelligent Mechatronics (2011), pp.560-565.

Jindai, M., Ota, S. and Sasaki, T., A Hug Behavior Generation Model Based on Analyses of Human Behaviors for Hug Robot System, Proceeding of 1st International Conference on Design, Mechanical and Material Engineering (2016), pp.1-7.

Kouchi, M., Mochimaru, M., Iwasawa, H. and Mitani, S., Anthropometric Database for Japanese Population, Japanese Industrial Standards Center 1997-98 (AIST,MITI) (2000).

Nakanishi, J., Kuwamura, K., Minato, T., Nishio, S. and Ishiguro, H., Evoking Affection for a Communication Partner by a Robotic Communication Medium, Proceedings of The 1st International Conference on Human-Agent Interaction, Vol.3 (2013), pp.1-4.

Rani, P., Sarkar, N., and Smith, C. A., Affect-Sensitive Human-Robot Cooperation -Theory and Experiments, Proceedings of the 2003 IEEE International Conference on Robotica \& Automation (2003), pp.2382-2387.

Teh, J., K., S., Cheok, A., D., Peiris, R., L., Choi, Y., Thuong, V. and Lai, S. Huggy pajama: a mobile parent and child hugging communication system, Proceedings of the 7th International Conference on Interaction Design and Children (2008), pp.250-257.

Tsetserukou, D., HaptiHug: A Novel Haptic Display for Communication of Hug over a Distance, Proceedings of EuroHaptics 2010 (2010), pp.340-347.

Watanabe, T. and Miwa, Y., Duality of Embodiment and Support for Co-Creation in Hand Contact Improvisation, Journal of Advanced Mechanical Design, Systems, and Manufacturing, Vol.6, No.7 (2012), pp.1307-1318. 